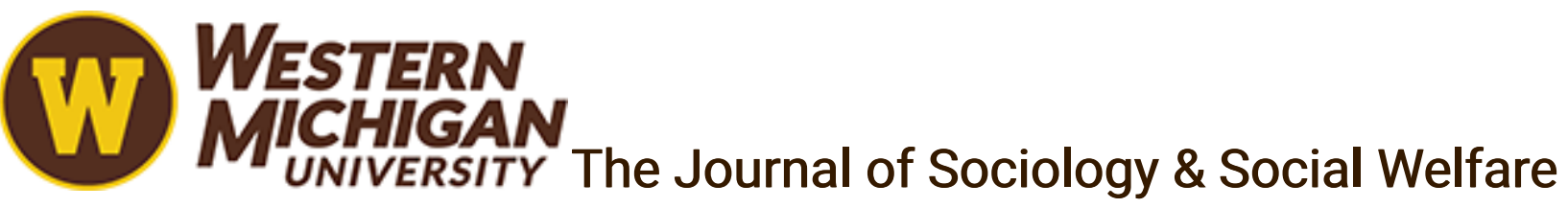

Volume 7

Issue 3 May

Article 4

May 1980

\title{
Organizational Structure and Professional Norms in an Alternative Health Care Setting: Physicians in Health Maintenance Organizations
}

Judith K. Barr

Rutgers University

Marcia K. Steinberg

Rider College

Follow this and additional works at: https://scholarworks.wmich.edu/jssw

Part of the Clinical and Medical Social Work Commons, Health Policy Commons, and the Social Work Commons

Recommended Citation

Barr, Judith K. and Steinberg, Marcia K. (1980) "Organizational Structure and Professional Norms in an Alternative Health Care Setting: Physicians in Health Maintenance Organizations," The Journal of Sociology \& Social Welfare: Vol. 7 : Iss. 3 , Article 4.

Available at: https://scholarworks.wmich.edu/jssw/vol7/iss3/4 
ORGANIZATIONAL STRUCTURE AND PROFESSIONAL NORMS IN AN ALTERNATIVE HEALTH CARE SETTING: PHYSICIANS IN HEALTH MAINTENANCE ORGANIZATIONS *

\author{
Judith K. Barr \\ Rutgers University, Newark
}

Marcia K. Steinberg

Rider college

The development of new organizational forms for the delivery of health and medical care in the U.S. includes health maintenance organizations (HMOs), designed to provide a set of comprehensive basic health services to a defined population for a fixed prepaid premium. As complex organizations, HMOs have the potential for limiting the autonomy of professionals working in them. This paper describes the legal requirements and organizational mechanisms under which physicians practice in HMOs and considers the potential for conflict between the organization and professional norms.

On the basis of document and interview data from nine HMOs, it appears that mechanisms developed to implement the mode of physician reimbursement and legal requirements for quality assurance and member grievance procedures do not limit physician autonomy in these HMOs. Variation was observed among the three organizational models: staff, group, and independent practice association.

${ }^{*}$ A version of this paper was presented at the Annual Meeting of the Society for the Study of Social Problems, Boston, August, 1979. The senior author appreciates the helpful comments of Louis H. Orzack and Ralph Larkin on an earlier draft. This research was supported in part by a grant from the Applied Social Research Coordinating Council, Rutgers University, Newark. 


\section{INTRODUCTION}

It has recently been argued that a variety of societal forces are combining to challenge the position of the medical profession and erode professional autonomy and control over conditions of practice. These forces include increasing bureaucratization and government intervention, as well as rising levels of consumer education and interest (Haug, 1976; Child and Schriesheim, 1978). One potential source of challenge lies in alternative ways of organizing medical practice and the delivery of services which have been developed as part of the response to problems in the health care system in the United States.

Among these alternative forms of health care delivery are health maintenance organizations (HMOs), designed to provide a set of comprehensive basic health services to a voluntarily enrolled population for a fixed, prepaid premium. Within this concept, a variety of organizational structures is possible, and different arrangements for the delivery of care have emerged with physicians providing services under varying modes of reimbursement. The purposes of this paper are to describe the organizational arrangements and legal requirements under which physicians practice in HMOs, to report empirical evidence about the implementation of these structural elements, and to consider the potential for strain between organizational requirements and professional norms.

\section{THEORETICAL BACKGROUND}

A considerable body of literature postulates a clash between the colleague control structures of professions and the system of hierarchical control characteristic of complex organizations (Blau and Scott, 1961; Etzioni, 1961; Thompson, 1961). Scott (1966:269) suggests that professionals may resist bureaucratic rules, reject bureaucratic standards, resist bureaucratic supervision and give limited, conditional loyalty to the organization.

Various accommodative mechanisms have been identified which limit strain between professional norms and bureaucratic requirements (Litwak, 1961; Scott, 1966). Barber (1963) notes the existence of differentiated role structures for carrying out professional work partially separated from the organization as a whole and differentiated 
authority structures in which professionals serve as administrators. Goss (1961) found that hospital clinic physicians accepted hierarchical authority exercised by physician administrators over administrative matters, while maintaining their individual authority over patient care activities within a framework of advisory relationships with physicians.

How professionals respond in organizational settings has been linked to various characteristics of the organization. In his comparative study of occupations, Hall (1968) found that professionalization and bureaucratization were inversely related so that perceptions of autonomy were negatively related to hierarchy of authority, division of labor, formal procedures, and impersonality. The generalization that the maintenance of professional norms varies with the level of bureaucratization of the setting has been supported in the health field. Engel (1969) observed that perceived autonomy among physicians varied with three types of bureaucratic settings: solo or small group practice (non-bureaucratic), a privately owned closed-panel medical organization (moderately bureaucratic), and a governmental medical organization (highly bureaucratic). Physicians in the moderately bureaucratic setting perceived the greatest autonomy with respect to their professional work; Engel concluded that there may be an optimal level of bureaucracy in which limits on autonomy are balanced by factors which facilitate professionals' goals.

Freidson (1970) has argued that autonomy is the core characteristic of a profession, that physicians have exclusive rights over medical practice, and that medical practice has been organized to facilitate physician autonomy and control.1 Larson (1977) has recently argued

$I_{\text {That these two concepts are empirically, as well as }}$ conceptually, distinct has been demonstrated in the work of Nathanson and Becker (1972). Following their distinction, autonomy is defined as freedom from non-professional determination and evaluation of work activities, whereas control is defined as influence over organizational policies and the work of non-professionals. 
that professions and organizations are both part of a process of rationalization of work; therefore, they may be seen as complementary rather than conflicting modes of organizing work. From another perspective, professions are seen as composed of segmented interest groups moving at different rates to maximize various "professional" characteristics (Bucher and Strauss, 1961). In this view, autonomy is not considered an attribute which necessarily accompanies the functioning of a professional (Roth, 1974). Rather, this concept is an important dimension regarding professionals in work settings which can be studied under varying conditions (Nathanson and Becker, 1972; Madison, et al., 1977).

From any of these perspectives, the extent of professional autonomy in bureaucratic settings can be considered problematic. As complex organizations, HMOs are one context in which this issue can be studied.

\section{HEALTH MAINTENANCE ORGANIZATIONS}

The term health maintenance organizations was first described in 1970 (Ellwood, et al., 1971). It incorporated group practice concepts and prepayment within a new structural form for the delivery of comprehensive ambulatory health care. HMOs were intended to address a variety of problems, including high costs and lack of accessibility, and to promote consumer accountability and quality of care. The Federal HMO Act (P.L. 93-222) of 1973 was the first effort to put this concept into law. According to the legislation, an HMO is an organized system for the delivery of a set of comprehensive health and medical services under a contractual arrangement with a voluntarily enrolled population for a fixed prepaid premium which is the HMO's major source of revenue.

In the 1976 amendments (P.L. 94-460) to the law, three organizational models are delineated, all operating under the prepayment mechanism but differentiated by practice site and mode of physician reimbursement (U.S. Department of Health, Education, and Welfare, 1977). These three models are:

(1) Staff: central facility; physicians are salaried employees of the HMO.

(2) Group: central facility; physicians are part of a medical group, partnership, or corporation reimbursed 
by salary or capitation ${ }^{2}$ through the group.

(3) IPA (Individual Practice Association): physicians practice in their private offices; physicians are part of partnership, corporation, or association which contracts with the HMO; and they are reimbursed individually on a fee-for-service basis through the medical group. ${ }^{3}$

The federal HMO law also sets guidelines and regulations which embody organizational requirements that may affect physicians and the way they practice. Ongoing quality assurance programs must be established to assure maintenance of standards and high quality in both the process and outcomes of care; such programs must ensure the HMO meets standards for hospitalization set up by physicians on a community basis. The law also requires that there be "meaningful" procedures for hearing and resolving grievances by HMO members, providing a mechanism for member complaints about services or other problems.

\section{SETTING AND METHODS}

Data were gathered from nine HMOs in 1978. They were studied because they include all operational HMOs in a single state and are subject to the same state law and regulations. These HMOs include the three models specified in the federal law. Five of the HMOs are federally qualified, and all are state certified. 4 These HMOs had been operational for one to five years. The number of physicians in staff and group model HMOs

${ }^{2}$ Capitation refers to a specified amount paid per enrollee for a specified period of time.

${ }^{3}$ In 1978, there were 203 HMOs in the U.S. serving more than seven million people. HMOs had been certified in 37 states, and 79 were federally qualified. Sixtyfour percent of the nation's HMOs were staff or group models (U.S. Department of Health, Education, and Welfare, 1978).

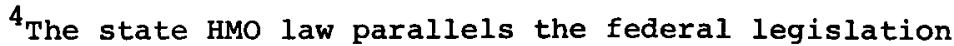
in requiring a quality review mechanism and a grievance procedure; it does not require that HMO members be on the board of directors of the HMO, as required by federal law. 
ranged from 12 to 42 , and additional specialists were available for referral in the community. Nearly 600 physicians belonged to IPAs, including both primary care physicians and specialists. The total number of enrollees ranged from approximately 1,000 to 22,000 members. The HMOs are located in urban, suburban, and semi-rural areas.

Sources of data are intensive, open-ended interviews with the HMO executive directors and medical directors, documents provided by the HMOs, and certificates of authority and annual reports filed with the state department of health. Information was collected in three areas in which organizational requirements may affect physician autonomy: reimbursement mechanisms, quality assurance programs, and grievance procedures. These three areas were selected because they are part of the HMO law and, thus, constitute legal requirements for the HMOs; also, these requirements may affect the physician's ability to set the financial value for his or her work, to make individual and independent medical decisions regarding patients, and to control response to patient complaints while being more vulnerable to patient demands.

As noted previously, there are findings which suggest that physician autonomy varies with the degree of bureaucratization of the setting, that organizational bureaucracy includes such characteristics as authority structure and formalization of rules and regulations, and that there are mechanisms by which professionals seek to maximize their autonomy under conditions of organizational constraints. Accordingly, data were gathered concerning who participates in establishing reimbursement, quality assurance, and grievance mechanisms; the extent of codification of the procedures; who has a role in carrying out the procedures; and the enforcement of standards.

\section{FINDINGS}

The evidence to be presented concerns the implementation of the HMO concept in three areas: (1) physician reimbursement mechanisms, (2) quality assurance programs, and (3) grievance procedures.

Reimbursement Mechanisms

In the staff model, salary negotiation with individual physicians is carried out by the executive director with the whole plan balance sheet in mind, or by the medical 
director with a budget to allocate among different physicians. In both cases, the budget is subject to review by the board which may include lay or nonprofessional members. Part-time physicians enter the same negotiation process but are reimbursed by capitation. In the group model, salary negotiation occurs among physicians in the group or between a physician and the representative of the medical group. These negotiations are subject to an agreement between the medical group and the HMO about capitation for the group. In both staff and group models, there were reports that negotiations took into account the "market value" differential among specialties. In the IPA model, the medical group contracts with the HMO for payment on a capitation basis; the medical group then reimburses individual physicians on a fee-for-service basis, using a scale based on the "usual and customary" charges of area physicians. Payment is an amount ranging from 80 to 90 percent of physician charges, with the remaining funds constituting a risk pool to cover excess costs to the plan for physicians' services.

\section{Peer Review and Quality Assurance}

As guidelines for practice and criteria for review of physician behavior are established, these may be written in the form of a physician handbook or protocols for practice. Five HMOs have written standards that are distributed to physicians. These may encompass administrative matters, including guidelines for record-keeping and rules for scheduling patient appointments, and may specify HMO services and the processes of monitoring how these services are delivered. The protocols also tend to set the tone for medical conduct by stating a general philosophy of medical practice ("...increase the effectiveness and quality of health care provided") or by listing goals of the organization (e.g., high quality care, continuing education for physicians, and patient education).

Standards for medical practice are empirically derived by physicians. The IPAs use standards developed by physician groups in the community (e.g., the county medical society); in the staff and group model plans, the standards are designed by the medical director and/or staff physicians or are derived from the practice patterns of the HMO physicians themselves. As emphasized in several interviews, the standards are intended to be general guidelines which reflect usual patterns of practice and are used to detect deviations from these pat- 
terns, not to specify step-by-step procedures for patient care. According to one HMO Certificate of Authority, "the standard does not define good or bad care. It is used to ascertain whether or not a chart or other performance is deviant."

Each HMO has a peer review system in which physicians examine their own records and those of their colleagues in three areas of medical practice: ambulatory care, hospitalization, and referral to specialists. The type of review activity, frequency of review, and person(s) doing the review vary in the different HMOs studied.

Ambulatory review. The initial screening of ambulatory medical records is performed by physicians in all except two of the plans. In all the HMOs, the quality assurance review process is carried out by one or more physicians. 5 Review of charts occurs at regular intervals or at the discretion of the medical director, and frequency of chart review varies from weekly to monthly. Generally, charts to be reviewed are selected at random, by disease category or by outcome. The review processes in these HMOs focus on a variety of items in the charts. In some plans there is a specific list of items to be reviewed, e.g., number of visits, duration of treatment, and prescriptions. As one medical director reported, in looking for deviations from accepted standards and prevailing patterns of practice, the physicians want to "locate weak areas of care."

When a deviation from the standard pattern is found, or if another problem is noted, decisions about what to do vary with the particular issue. In the interviews, executive directors and medical directors reported that physicians were not reprimanded as if performance had been judged inadequate; rather the emphasis is on counseling, educating, and advising physicians. Where the problem is judged to be that of an individual physician, usually the medical director informally brings it to his or her attention. For other problems, the medical direc-

${ }^{5}$ In two plans, nonphysicians also participate in the quality assurance review process; these include nurses, medical records personnel, and the center director. This participation appears to be an administrative role only, with no input in setting standards. 
tor may send a letter discussing the issue to all the physicians rather than singling out a particular physician.

The review process is also used as a basis for revising existing standards of practice within the plan. The review of an individual physician's chart may prompt a review of the records of all physicians in a specific area of practice. If patterns of practice behavior are uncovered that appear to be inappropriate, the group revises the standards. Thus, consensus develops as part of the review process in regard to standards for practice and specific problems which are raised in the record review.

While all the HMOs are required to have ambulatory review procedures, the extent to which these procedures are formalized as rules of the organization varies. As suggested by Aiken and Hage (1966), two aspects are important to consider: the degree of codification of the rules (as in the written physician protocols) and supervision in adherence to these rules (through the quality assurance and review mechanisms). Physicians participate in both of these organizational processes. It is physicians who set the framework within which the ambulatory review process takes place by developing the standards used to judge physician practice, and they are the exclusive participants in seven of the HMOs studied.

Referrals to specialists. Some type of authorization is required for all referrals. In-house referrals must be authorized by the primary care physicians; that is, there are no self-referrals by patients. Referrals to specialists outside the HMO must be countersigned, in most plans by the medical director. This system provides a formal mechanism for review of physician's work in regard to appropriateness of referrals. In one instance, a medical director found that a pediatrician had made an unusual number of referrals to the orthopedic specialist because he felt unsure treating these cases. The pediatrician was sent for a special course in orthopedics, part of the rationale being that it is cheaper to keep simple cases in-house than pay a specialist for referrals.

Referrals to hospitals. A retrospective review of hospitalizations for enrollees exists in all the HMOs. Post-admission review of hospital records and authoriza- 
tion for the admission is made by a nurse or other nonphysician who reviews the admission for appropriateness and length of stay according to standards developed by a designated organization of physicians in the community. Subsequent concurrent reviews are conducted at specified intervals to authorize continued hospitalization. This review process is similar to that for physicians in private practice. According to legislation which establishes Professional Standards Review Organizations (PSROs), postadmission reviews of hospitalizations for Medicaid and Medicare patients must be conducted for appropriateness of service and length of stay (Goran, et al., 1975). Some states have enacted legislation to permit third-party payers access to hospital utilization review data, widening the review process for physicians in private practice.

\section{Grievance Procedures}

Consistent with federal and state regulations, there are formal grievance procedures developed by each health plan which outline the steps an enrollee may take to register a complaint. A written statement of procedures is provided for the new member; it may be part of the enrollee contract, contained in a member handbook, or available as a separate handout.

In each of the HMOs, the first step is to give the complaint, in written or oral form, to a designated nonphysician staff member, such as a member services coordinator, who attempts to resolve the complaint. The next steps in the process vary considerably among the HMOs; complaints are referred to the medical director, the center administrator, the executive director, the appropriate department head, or a review committee which may include physicians and/or board members. Consumer representatives are involved at this interim stage in five of the plans. The HMO Board of Directors has final authority in four of the plans; four have outside arbitration as the final step, and one has a joint committee as the final arbiter. In three HMOs, at least one consumer must be part of the final appeals body. Generally, the medical profession is represented in the grievance process through the medical director. In half of the plans, the medical director has a decision-making role in the formal process; and physicians have a formal role in the grievance structure in three plans.

Although the grievance system establishes a formal 
mechanism for patient complaints, in their operation, these structures do not appear to have impinged on physician autonomy. In each HMO, the staff has established and maintained a distinction between administrative and medical matters, usually through informal understandings. Very few complaints about individual physicians were reported. Whenever questions regarding medical practice arise, they are referred to the medical director or the physician department chief for review and resolution. Most complaints have concerned matters respondents identified as "process" issues, such as the availability of after-hours services, appointment waiting times, and services to which enrollees believe they are entitled. These complaints are considered administrative matters and are referred to the executive director, the center administrator, or the appropriate department head. Interviews indicate that most enrollee grievances are resolved informally, by bringing the complaint to the attention of the physician or other staff person.

\section{DISCUSSION}

Given the requirements for reimbursement levels, quality review, and grievance procedures, it might be expected that in settings such as HMOs, the professional norm of autonomy would be in conflict with bureaucratic structures. Data from documents and administrator interviews in nine HMOs indicate that physician autonomy does not appear to be limited by the way in which these structural mechanisms are implemented.

Although forms of reimbursement vary, physicians participate to some extent in how they operate. Individual physician income in staff and group models is set with the active involvement of the physician. Salary and capitation rates are determined through a process of negotiation rather than through use of fixed, predetermined scales. In most cases, the physician negotiates with the medical director rather than the executive director. Rates for physician services in the IPAs are based on prevailing fees in the community. In all HMOs, physicians are involved in setting income levels, permitting the profession to maintain a degree of autonomy in this aspect of practice.

Quality assurance systems represent one form of differentiated role structure (Barber, 1963) which permits 
physicians to maintain autonomy and limits potential strain between organizational requirements and professional norms. In these HMOs, there is systematized accountability to the medical profession; physicians set the standards for review and carry out the review process. What constitutes autonomy for the profession may not constitute autonomy for the individual physician. Quality assurance procedures subject physicians' work to a review process. Furthermore, the individual physician may be told to alter aspects of medical work which other physicians decide do not meet group standards. The individual physician may respond to this as an intrusion into his practice and an infringement of autonomy, or as an opportunity to improve work through peer discussion.

Through the grievance system in HMOs, physician behavior that might not be subject to challenge by patients in a solo, fee-for-service practice is potentially subject to review. Members have contractual rights with the organization for a set of services, and they can be expected to make demands which may represent a challenge to physicians (Goss, et al., 1977; Freidson, 1975). It is the grievance procedure which provides a vehicle for patient complaints. In these HMOs, few complaints have been raised about individual physicians. Those grievances that have been voiced were settled informally, after referral to the medical director, without going beyond this first step in the grievance procedure. It seems that in the daily routines of the HMOs, physician autonomy has not been challenged by members.

Comparing these structural features among the three types of HMOs, there is variation (1) in the relationship of professionals to the organization, and (2) in the degree of formalization of procedures and the formal role for different organizational participants. As Hall (1968) and Engel (1969) have shown, such differences, as components of bureaucratization, may be related to levels of autonomy.

Considering the relationship of professionals to the organization, 6 the IPA is distinguished from staff and

${ }^{6}$ In all the HMOs, a physician serves in the administrative role of medical director; this position provides a differentiated authority structure (Barber, 1963), a professional authority structure to which the physicians are subject. 
group models in several respects. IPA physicians may not practice in a group setting; only a portion of their patients are HMO enrollees; they are paid on the usual feefor-service basis; and as HMO physicians they are members of a physicians' group which contracts with the HMO for the provision of member services. This group has a formal relationship to the plan, and mutual obligations between the HMO and the physician members are detailed. Compared to physicians in staff and group models, the IPA physicians are more similar to the "ideal type" physician in terms of practice setting and reimbursement arrangement. They are similar to physicians in the group model in having a formal contractual relation with the HMO for the provision of member services as part of a physician group.

There is evidence of a continuum of organizational types based on the dimension of degree of formalization of procedures and rules for quality assurance and member grievance. The IPA models appear to be the most formalized and structured; the group model is a mixed type; and the staff model is the least formalized. In the IPA models, there are written protocols for practice and a medical group review committee for quality assurance. The member grievance system includes a formal role for the medical director and for physicians, and consumers are included in the final appeals procedure within the plan. While some of the staff model HMOs have written protocols for practice and a formal role for the medical director in the grievance system, none has a formal role for physicians, and none requires that consumers be part of the final appeals body within the plan. The group model HMOs are divided, paralleling the structures in either the IPA or staff models.

From the data on structure in these HMOs, it appears that the IPA model offers greater opportunity for maintaining autonomy of the profession as a whole than do other models. In one case, the local pSRO is closely associated with the medical group and sets the review standards for the IPA, thus consolidating the position of the profession. Because the IPAs appear to be more formalized and more structured than the other models, with physicians establishing and implementing the structure, it is likely that IPA physicians are less subject to controls from outside the profession.

The staff models in this study tend to operate on a 
more informal basis with fewer prescribed situations in which physicians participate. Perhaps because the physicians practice in a central location, communication and observability may facilitate the development of informal norms which support individual physician autonomy. Physicians in staff and group models, while subject to the structural requirements of the HMO, appear to have the opportunity to participate in implementing these requirements, and in so doing to negotiate their relationship to the organization, thus maintaining autonomy. Another possibility is that individual professionals may vary in their conformity to professional and bureaucratic norms; for example, the HMO staff physicians may value organizational requirements set forth to promote patient interests and feel that these do not limit autonomy with regard to practice.

\section{IMPLICATIONS AND CONCLUSIONS}

The bureaucratic structure of HMOs provides a setting in which professional norms may be challenged. From the available evidence, it appears that physician autonomy may not be diminished by the structural mechanisms for physician reimbursement, quality assurance, and member grievances. Rather, the medical profession, as well as individual physicians, are involved in defining and implementing these mechanisms. These requirements were established by the HMO legislation as part of an attempt to rationalize the health care system and make it more responsive to consumers by providing accessible high quality care at a reasonable cost, and in so doing to focus on prevention of illness and maintenance of health.

The data on HMO organizational characteristics reported in this paper suggest several implications for these broad goals. The process of negotiating their own income is one way that physicians may become more aware of the financial concerns of the HMO, and they may take these into account in decision-making about individual treatments. The different hospitalization rates among staff, group, and IPA models (U.S. Department of Health, Education, and Welfare, 1978) may to some degree reflect differential physician participation in financial issues. on the other hand, physician pressures for higher reimbursement may lead to increased costs for the HMO.

To the extent that practice standards become codified 
as part of quality assurance requirements, there may be increased rationalization of medical knowledge. In the HMOs studied, there were variations in the extent of codification and the rules for enforcement. Physicians maintained autonomy in both establishing and carrying out the quality assurance programs, and no nonphysicians or consumers participated in a meaningful way in assuring quality of care. These observations suggest that while medical knowledge may become more rationalized and physician accountability more systematized, medical care remains the responsibility of physicians and the medical profession. It has been suggested that in the face of policies intended to alter practice patterns, physicians may maintain dominance while becoming more routinely accountable (Goss, et al., 1977).

The grievance process in HMOs consists of detailed procedures which HMO members are informed about and encouraged to use. This complaint structure is an entry point for the consumers of health services to express objections when they are dissatisfied with organizational practices and the way services are provided. Yet, in these HMOs, members have few complaints and those that are voiced are easily resolved. Whether HMO members are more satisfied consumers cannot be judged from these data. It may be that HMOs are more responsive to consumer interests, or that given a structured opportunity to register specific complaints, consumers are reluctant to do so and need more time to become familiar with this mechanism.

The more rapid rate of growth of IPA models (U.S. Department of Health, Education, and Welfare, 1978:10) suggests that physicians may be seeking ways to maintain autonomy of the medical profession in an increasingly bureaucratic environment. This model allows for decentralized practice sites and traditional practice arrangements (either solo or group practice) while changing the method of financing to a prepayment mechanism. It is not clear whether this model will substantially lower costs and improve quality as well as physician accountability to the public.

Generalizations from these data are limited by the number of HMOs and the geographic area of the country in which they are located. It is suggested that through physician participation in organizational mechanisms, the potential for clash between bureaucratic requirements and 
the professional norm of autonomy may be reduced. There is differential participation by physicians in negotiating salary and reimbursement, as well as in the establishment and operation of quality assurance mechanisms and member grievance procedures. These variations suggest that individual physician participation in these mechanisms will be related to perceptions of autonomy, and that physician responses will vary in the different HMO models. To study these issues, evidence is needed about physicians' involvement in organizational decisionmaking and their perceptions of autonomy under varying organizational conditions.

\section{REFERENCES}

Aiken, Michael and Jerald Hage

1966 "Organizational Alienation: A Comparative Analysis." American Sociological Review 31 (August): 497-507.

Barber, Bernara

1963 "Some Problems in the Sociology of the Professions." Daedalus 92 (Fall):669-688.

Blau, Peter M. and W. Richard Scott

1961 Formal Organizations. San Francisco: Chandler Publishing Company.

Bucher, Rue and Anselm Strauss

1961 "Professions in Process." American Journal of Sociology 66 (January) : 325-334.

Child, John and Janet Schriescheim

1978 "Changes in the Social Position of Professional Occupations." Paper presented at the American Sociological Association Annual Meeting, San Francisco, September.

Ellwood, Paul M., Jr., Nancy N. Anderson, James E. Billings, Rick J. Carlson, Earl J. Hoagberg, and Walter McClure

1971 "Health Maintenance Strategy." Medical Care 9 (May-June) : 291-298. 


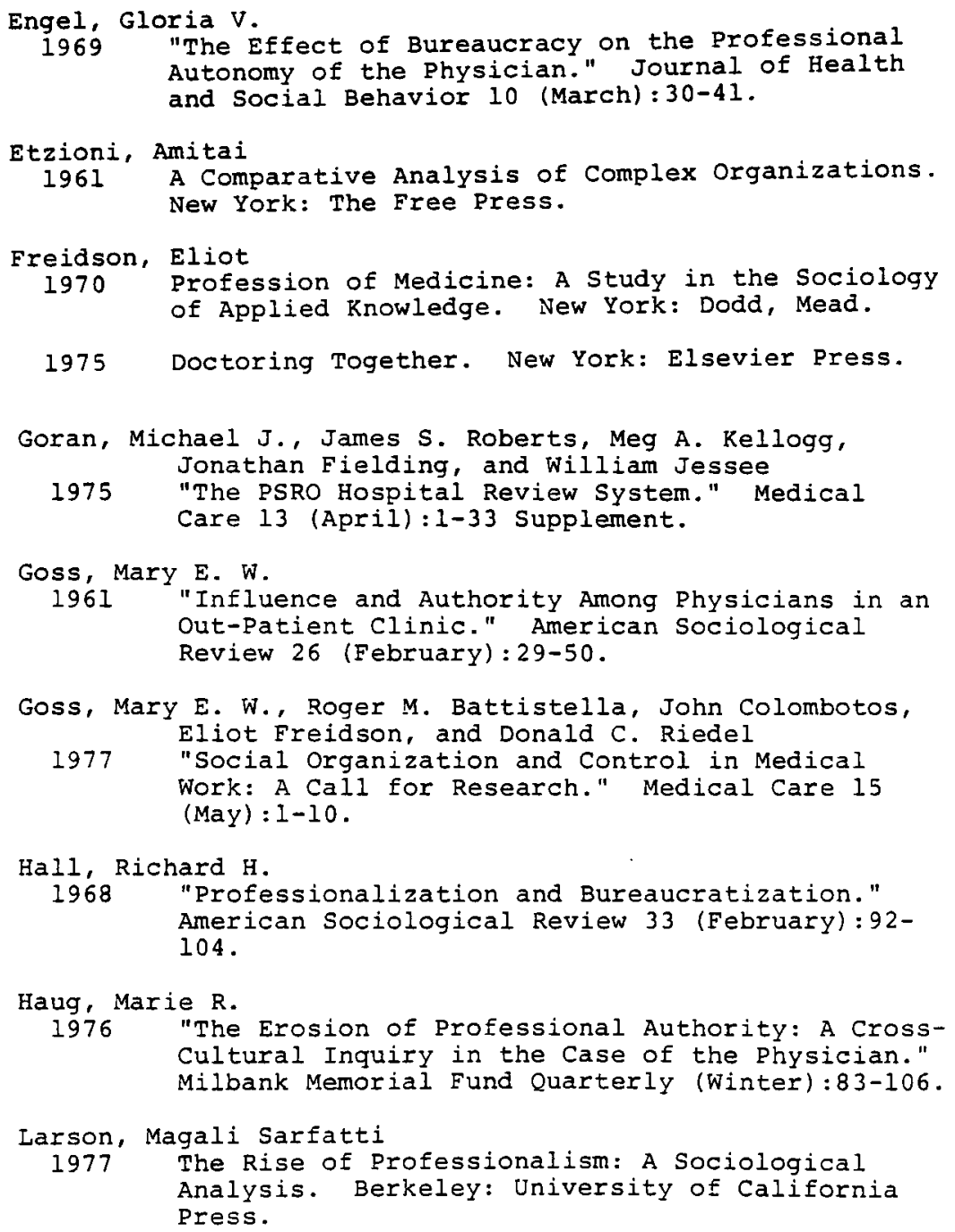




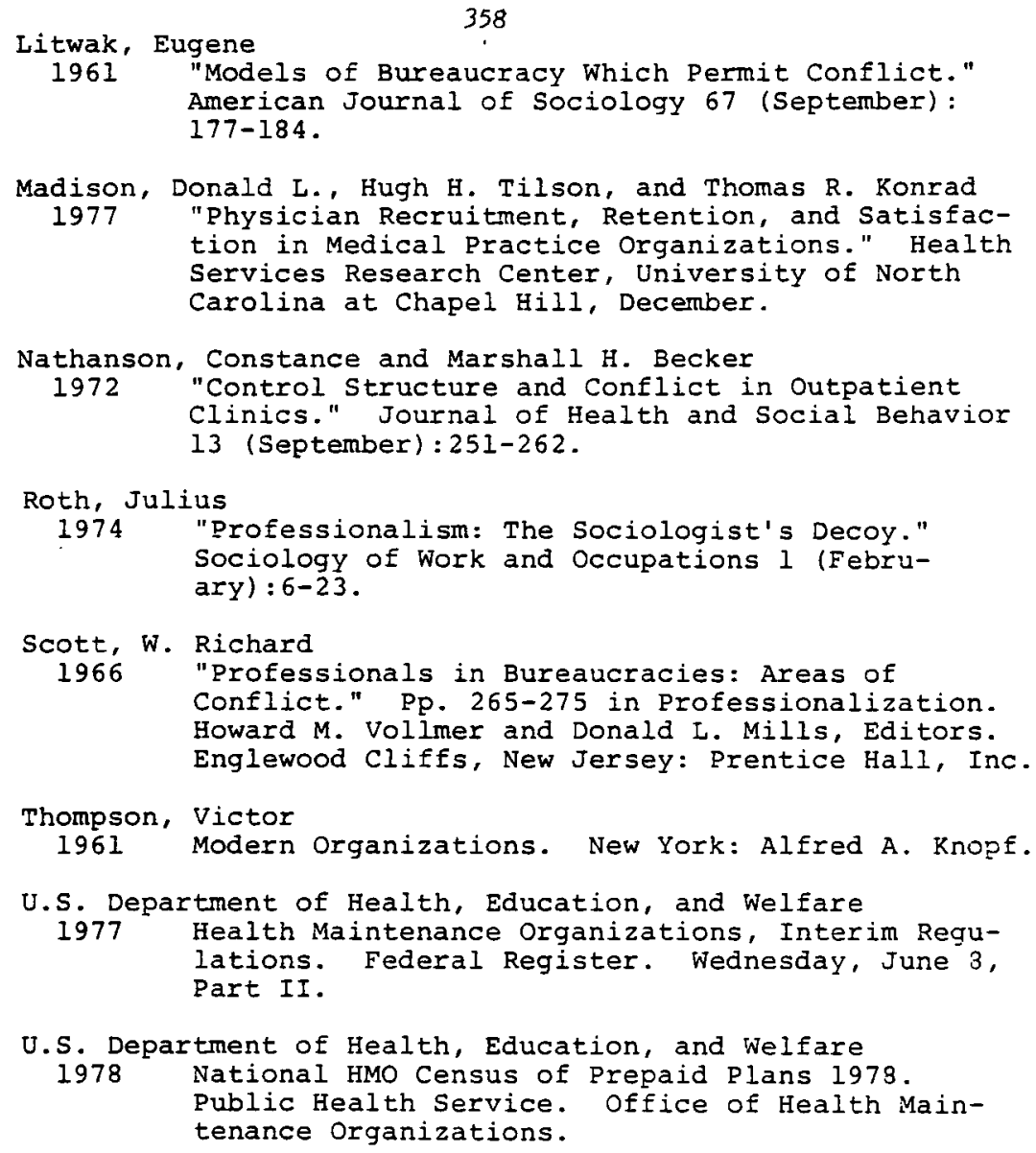

Madison, Donald L., Hugh H. Tilson, and Thomas R. Konrad

1977 "Physician Recruitment, Retention, and Satisfaction in Medical Practice organizations." Health Services Research Center, University of North Carolina at Chapel Hill, December.

Nathanson, Constance and Marshall H. Becker

1972 "Control Structure and Conflict in Outpatient Clinics." Journal of Health and Social Behavior 13 (September) :251-262.

Roth, Julius

1974 "Professionalism: The Sociologist's Decoy." Sociology of Work and Occupations 1 (February) : 6-23.

Scott, w. Richard

1966 "Professionals in Bureaucracies: Areas of Conflict." Pp. 265-275 in Professionalization. Howard M. Vollmer and Donald L. Mills, Editors. Englewood Cliffs, New Jersey: Prentice Hall, Inc.

Thompson, Victor

1961 Modern Organizations. New York: Alfred A. Knopf.

U.S. Department of Health, Education, and Welfare

1977 Health Maintenance Organizations, Interim Regulations. Federal Register. Wednesday, June 3 , Part II.

U.S. Department of Health, Education, and Welfare 1978 National HMO Census of Prepaid Plans 1973. Public Health Service. Office of Health Maintenance Organizations. 